\title{
Development of SYBR green I based one-step real-time RT-PCR assay for the detection and differentiation of very virulent and classical strains of infectious bursal disease virus
}

\begin{abstract}
A SYBR Green I based one-step real-time reverse transcriptase polymerase chain reaction was developed for the detection and differentiation of very virulent (vv) and classical strains of infectious bursal disease virus (IBDV). The assay showed high PCR efficiency $>93 \%$ and high reproducibility with coefficient of variation less than $0.5 \%$. Whentested on characterized IBDVstrains, the very virulent and classical-specific primers detected accurately only vvIBDV and classical IBDV strains, respectively. The diagnostic efficacy of the assay was also tested on 140 bursal samples from experimental infection and 37 bursal samples from cases suspected of IBD. The assay was able to detect IBDV from bursal samples collected at days 3 and 5 post-infection with the vvIBDV strain UPM94/273 and the classical IBDV strain D78. The assay was also able to detect bursal samples infected dually with D78 and UPM94/273. The melting temperature values of the amplification products from the classical and very virulent viral infection were statistically significant $(\mathrm{P}<0.05)$. The specificity of the assay for detecting IBDV from suspected cases was confirmed by sequence analysis of the VP2 gene. The assay showed high sensitivity since bursal samples whichwere negative for IBDV were confirmed by virus isolation and PCR amplification. Hence, the new assay offers an attractive method for rapid detection of strains of IBDV.
\end{abstract}

Keyword: Infectious bursal disease virus,Very virulent strain, Classical strain,VP4 gene,1 SYBR Green 1 based real-time, polymerase chain reaction 